\title{
Design of on-line PH monitoring system based on ZigBee technology
}

\author{
Laidi Wang ${ }^{\mathrm{a}}$ and Xiuli Zhang \\ College of Electronic and Information Engineering , Zhejiang Wanli University, Ningbo 315100, Zhejiang, China
}

\begin{abstract}
The $\mathrm{pH}$ of water is very important to human health and aquatic products breeding. Traditional PH detection technology cannot meet the requirements of real-time, dynamic and efficient monitoring, this paper designs an on-line PH monitoring system based on ZigBee technology. The system is based on the ZigBee protocol, using the SoC chip CC2530 which is completely compatible with protocol 2007 and manufactured by the TI company, DHT11 temperature and humidity sensor and the PH composite electrode, for real-time acquisition and monitoring of $\mathrm{PH}$ value. System also uses a linear regression algorithm to deal with $\mathrm{PH}$ value, in order to reduce the influence of temperature on $\mathrm{PH}$ measurement precision. Comparative measurement datum after processing show that we eliminate the influence of temperature on the PH monitoring with temperature compensation model.
\end{abstract}

Keywords: ZigBee; wireless sensor network (WSN); CC2530; PH monitoring.

\section{Introduction}

$\mathrm{PH}$ value include chemical analysis, test paper analysis and potentiometric analysis. Both of the former cannot realize real-time online measurement of $\mathrm{PH}$ value, and the potentiometric analysis method usually use composite electrode sensor, this kind of instrument is small, easy to use and the measurement data are accurate and reliable. Combined with wireless sensor network technology which has been concerned in the current international, can real-time monitoring, awareness and acquisition of all kinds of water environment information, so as to take timely and effective measures to control water quality, to guarantee the quality of water, reduce the labor intensity of workers have important practical significance. This paper designs the wireless sensor network water quality monitoring system based on ZigBee technology, this system has low operating cost, low power consumption, high reliability and broad application prospects.

\section{ZigBee technology}

ZigBee is the standard of a series of communication protocols for wireless communication which defines the short distance, low data transmission rate[2]. Its characteristics consist of high reliability, low cost, low power consumption, high safety and low data rate and mainly used for short distance wireless control system to transfer a small amount of control information. The working frequency of wireless network based on ZigBee is $868 \mathrm{MHZ}$ (Europe), $915 \mathrm{MHZ}$ (USA), $2.4 \mathrm{GHz}$ (global), and the

\footnotetext{
${ }^{a}$ Corresponding author : 1983210500@qq.com
}

(C) 2016. The authors - Published by Atlantis Press 
maximum data transmission rate is 250kbps. ZigBee protocol is on the basis of the OSI reference model, combined with the characteristics of wireless network layered, IEEEE 802.15.4 only defines the ZigBee physical layer (PHY) and media access control layer (MAC), ZigBee protocol defines the network (NWK) layer, application support (APS) and the application layer (APL) data transmission specification [3].

\section{The overall design of PH monitoring system}

The overall framework of the PH water quality monitoring system is shown in Figure 1. A plurality of data acquisition nodes are distributed in the monitored area, and each node form a self-organization network by adopting the ZigBee protocol, each node collects the $\mathrm{pH}$ value and temperature regularly and sends it to the data sink node through the ZigBee network. Finally, data sink node transmits the data to the monitoring center computer in real-time through the serial port. According to the monitoring center receives the data to calculate the $\mathrm{pH}$ value of each acquisition node.

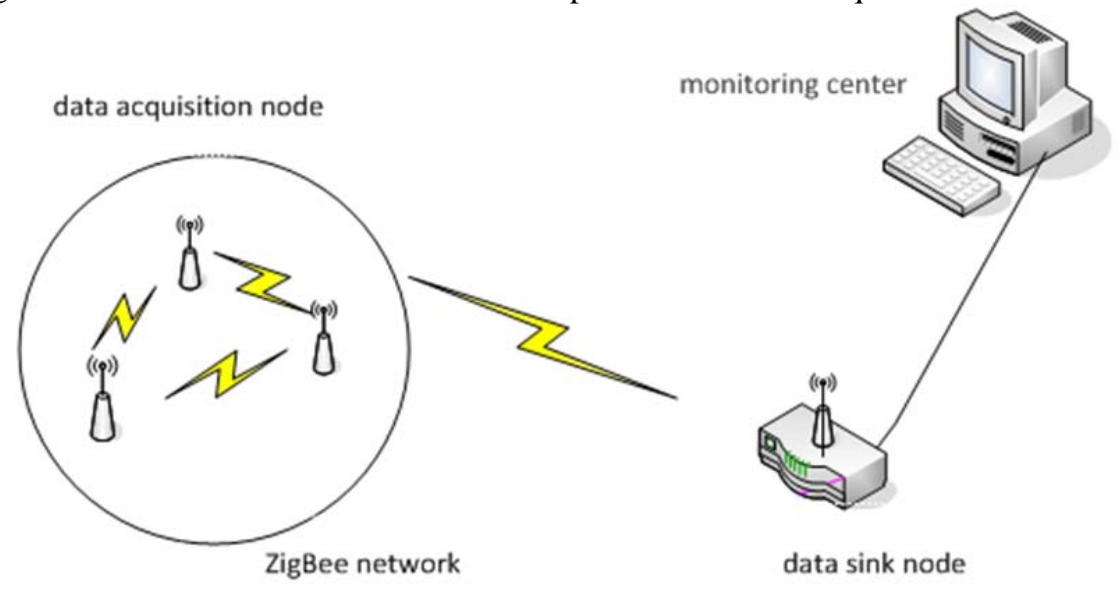

Figure 1. The framework of PH monitoring system.

\section{The hardware design of the system}

The hardware of $\mathrm{PH}$ monitoring system is composed of ZigBee network and gathering node, and ZigBee network is composed of many terminal acquisition node, the main control part of the terminal acquisition node and coordinator node adopts the same CC2530 master control chip. Every terminal acquisition node includes master control module, $\mathrm{PH}$ acquisition module, the temperature and humidity acquisition module and A/D conversion module.

\subsection{Master control module design}

Master control module includes RF chip, antenna, power supply, communication interface and other parts, RF chip select CC2530 as the core chip. CC2530 has a high performance $2.4 \mathrm{GHz}$ direct sequence spread spectrum radio frequency transceiver and a high performance, low cost 8051 microcontroller core, to meet the requirements of low cost, low power consumption of wireless sensor networks, and has a strong anti-interference ability, won't make the transmission of data inaccurate or distortion [4].Master control module circuit is shown in figure 2. 


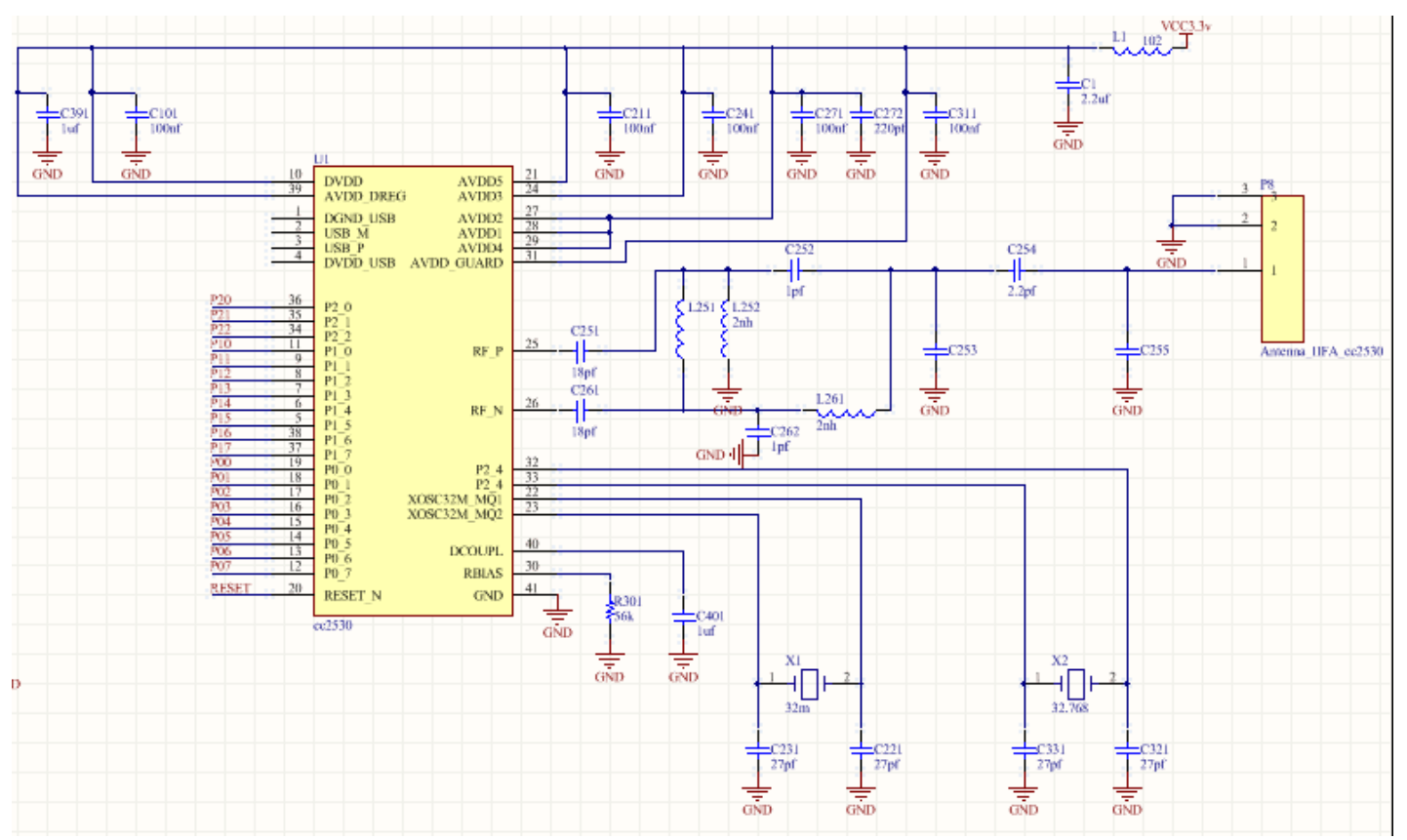

Figure 2. CC2530 master control module circuit diagram.

\section{2 $\mathrm{PH}$ acquisition module}

$\mathrm{PH}$ value is a sign of acid and alkali solution, national standards for the discharge of the $\mathrm{pH}$ value between 6 9. $\mathrm{PH}$ value is measured by the $\mathrm{PH}$ composite electrode, the $\mathrm{pH}$ value of the water can be calculated by the electric potential which is produced by the different of the acidity and alkalinity of the water. Due to PH composite electrode is influenced by its high resistance, so the output signal is small, in order to satisfy the input range of the $\mathrm{A} / \mathrm{D}$ analog digital conversion circuit, the output signal must be amplified. As shown in figure 3, the op-amp differential amplifier circuit is used to achieve values in the range of $0 \sim 3.3 \mathrm{~V}$, and improve the accuracy of the data acquisition. The output of the circuit is connected to the P0_7 pin of the CC2530 master control chip.

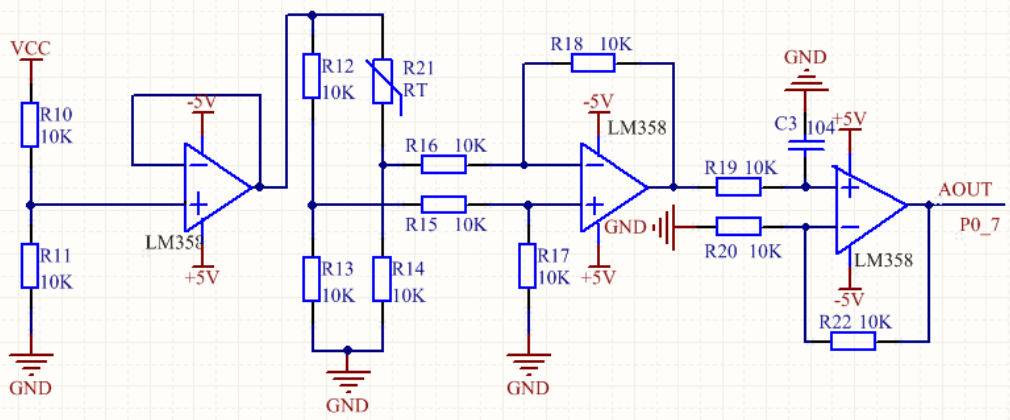

Figure 3. PH measurement adjustment circuit.

\subsection{Temperature and humidity acquisition module}

Temperature and humidity acquisition are realized by DHT11 sensor. DHT11 is a kind of temperature and humidity sensor which has been calibrated digital signal output, and the sensor has high reliability and long-term stability. As shown in figure 4, the P11 pin is used for the communication and 
synchronization between the microcontroller and DHT11, the two sides are using a single bus data format for information transmission, a communication time 4ms. DHT11 will not take the initiative to collect information, it will begin by receiving from the terminal device to start the signal. After finish collecting the data, it will transition to the low speed mode.

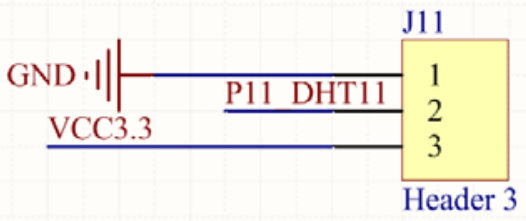

Figure 4. Temperature and humidity acquisition circuit.

\subsection{A/D conversion module}

The PH sensor in this system needs to change the analog signal to digital signal. CC2530 has an analog multiplexer with 8 configurable channels. ADC supports 14 bit analog digital conversion, and has as many as 12 bits of valid digit. Registers are ADCL (storage low part of the ADC conversion results), $\mathrm{ADCH}$ (storage high part of the ADC conversion results), ADCCON1, ADCCON2 and ADCCON3 (the three control registers are used for ADC configuration state, conversion sequence, sequence of execution mode conversion, reference voltage, sampling rate selection etc.), the ADCCON1.EOC bit is the status bit, which is 1 when the conversion is finished; the ADCCON1.ST bit is used to start a AD conversion; the ADCCON2.SREF is used to select the reference voltage. The system chooses the $3.3 \mathrm{~V}$ as the reference voltage, and uses the 12 bit conversion precision, that is to say, 3.3V corresponds to 4096 of the conversion value, the complete sign of conversion can be queried by scanning or interrupt the way.

\section{The system software design}

Software design of the system is realized on the basis of the hardware platform, and it is an important part of the system function. The system software includes 3 parts, ZigBee network software, terminal node software and coordinator software. Software development environment is IAR Embedded Workbench (EW), its C cross compiler is a complete, stable and Easy to use development tools. At present, it can support at least 35 kinds of ARM microprocessor architecture. It has the characteristics of a fully compatible with standard C language, the corresponding chip program rate and internal optimizer, memory mode selection and efficient Pro Mable. The use of the debugger is SmartRF04EB which is recommended by TI, and its program download speeds up to $150 \mathrm{~Kb} / \mathrm{s}$, and support the TI company's production with 80C51 kernel of ZigBee chip, can be seamless connection with IAR integrated environment[5].

\subsection{ZigBee network software design}

ZigBee network protocol stack is Z-Stack, it's clear structure, easy to transplant, hierarchical is able to run in a multi tasking environment in the code. The whole Z-Stack uses a layered software architecture, including the PHY layer, MAC layer, NWK layer, APS layer, APL layer, security layer and operating system abstraction layer. The main function in ZMain, mainly includes two parts, namely the system initialization process and implementation of operating system entities. Among them, the "osal start system()" is the operating system entities code, it is a dead cycle, and continuous query each task in whether there is any event, if there is an event, through comparing the priority, executive corresponding processing function; If no event occurs, then continue to query the next task, so keep the cycle. 


\subsection{Terminal node software design}

The terminal node is responsible for data collection, processing and communication of the collected data. After start-up is initialized first, then choose communication channels, automatic scanning network and join. After the success of the network, the terminal will be received from the coordinator of the acquisition request, and then start the timer capture, nodes receive timing acquisition news(WORK_TIME_EVT), to complete the corresponding data collection, and then by calling the function AF_DataRequest () to complete the transmission of data. The terminal sends the data to the coordinator in the form of unicast, and the coordinator receives the information in the form of broadcast. The program flow chart is shown in figure 5.

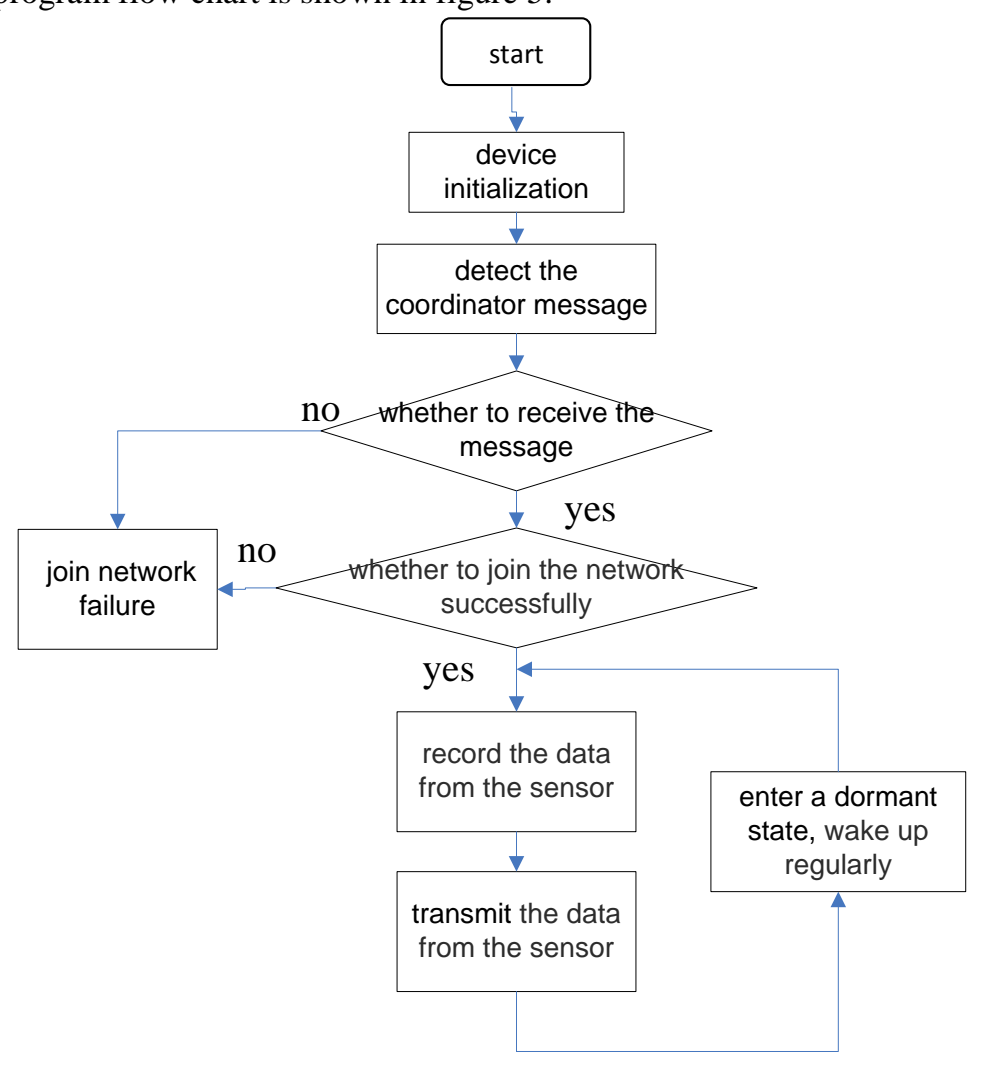

Figure 5. Flow chart of terminal node.

\subsection{The coordinator software design}

Coordinator is quite important for network, on the one hand ,it should be responsible for the formation of the Internet and the management, on the other hand also receive data from the terminal node, and make them converge to the monitoring center. After the start, first of all, I/O port, serial port and RF system initialization, and then select the corresponding channel to form a network, Then enter the OSAL scheduling system, OSAL will regularly scan the message status table to see if the new message is received, if have, OSAL will call the corresponding processing program. The news which need to deal with consists of RF receive messages(AF_INCOMING_MSG_CMD) and messages from the serial port control command (MT_SYS_CONTROL_MSG), This part of the software mainly includes device initialization, coordinator network, node join network, wireless data transceiver and processing procedures, the program flow chart is shown in figure 6. 


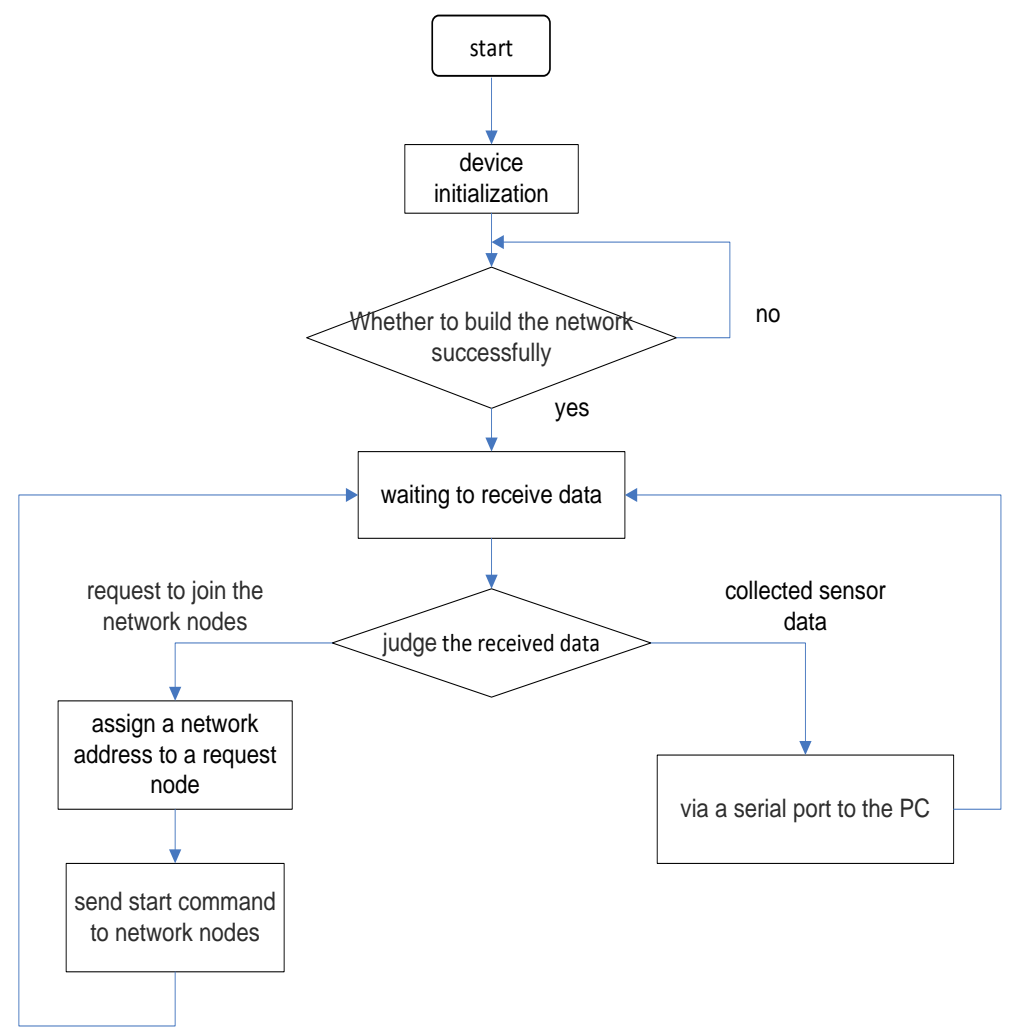

Figure 6. Flow chart of Coordinator.

\section{The data processing method}

Because of the monitoring system installed in damp environment for a long time, temperature on the $\mathrm{PH}$ measurement results had a greater influence. In order to reduce the influence of temperature on the output signal of $\mathrm{PH}$ sensor, after extracting the original data from the sensor, the temperature compensation is needed to improve the accuracy of PH detection. From the literature 6 we can see that when the temperature is kept constant, the $\mathrm{pH}$ value and the $\mathrm{PH}$ composite electrode potential basically meet the linear relationship. A linear regression analysis method is used to compensate the temperature of the sensor, assuming that the output voltage of the sensor is $x$, the theoretical value of the liquid $\mathrm{PH}$ is $y$, to make regression analysis for $y=a x+b$, in which $a$ and $b$ are the regression coefficient[6-7].

In order to determine the regression coefficients $a$ and $b$, least square method is usually used to achieve the minimum value of $Q=\sum_{i=0}^{n-1}\left[y_{i}-\left(a x_{i}+b\right)\right]^{2}$. According to the principle of extreme value, $a$ and $b$ satisfy the following equation:

$$
\begin{aligned}
& \frac{\partial Q}{\partial a}=2 \sum_{i=0}^{n-1}\left[y_{i}-\left(a x_{i}+b\right)\right]\left(-x_{i}\right)=0 \\
& \frac{\partial Q}{\partial b}=2 \sum_{i=0}^{n-1}\left[y_{i}-\left(a x_{i}+b\right)\right](-1)=0
\end{aligned}
$$




$$
\text { Solution } a=\frac{\sum_{i=0}^{n-1}\left(x_{i}-\bar{x}\right)\left(y_{i}-\bar{y}\right)}{\sum_{i=0}^{n-1}\left(x_{i}-\bar{x}\right)^{2}} \quad b=\bar{y}-a \bar{x} \text { in which } \bar{x}=\sum_{i=0}^{n-1} x_{i} / n \quad \bar{y}=\sum_{i=0}^{n-1} y_{i} / n
$$

The regression coefficients $a$ and $b$ of the three kinds of temperature are calculated by monadic linear regression analysis method, and the results are shown in Table 1.

Table 1. Values of regression coefficient a and $b$ in three kinds of temperature.

\begin{tabular}{|c|c|c|}
\hline Temperature $/{ }^{\circ} \mathrm{C}$ & Slope (a) & Constant (b) \\
\hline 10 & -5.6254 & 27.7163 \\
\hline 20 & -5.6351 & 27.7851 \\
\hline 30 & -5.6456 & 27.8566 \\
\hline
\end{tabular}

Analysis of the change of the slope a in the table, temperature $10{ }^{\circ} \mathrm{C}$ per change, a value changes are $0.0097,0.0105$, $b$ value changes are $0.0688,0.0715$, the average value of a and $b$ are 0.0101 , 0.07015 , from which can calculate the slope change rate of the system is $\Delta \mathrm{a}=0.00101 \mathrm{~V} /{ }^{\circ} \mathrm{C}(\mathrm{PH})$, the rate of change of $\mathrm{b}$ is $\Delta \mathrm{b}=0.007015 /{ }^{\circ} \mathrm{C}$. Finally, the temperature compensation model of $\mathrm{PH}$ sensor is obtained: $P H=-(5.6153+0.00101 T) E+0.007015 T+27.7462$

\section{Experimental test results analysis}

In the laboratory condition, I selected three concentrations of solvents $(4.00,6.86,9.18)$ and measured them. As shown in table 2, Compared with measurement of $\mathrm{pH}$ value and standard value, it can be seen that the error is very small. It shows that the measurement accuracy is better, and it is proved that the linear regression analysis is effective when the temperature compensation of the $\mathrm{PH}$ sensor is processed.

Table 2. Experimental results.

\begin{tabular}{|c|c|c|c|c|c|}
\hline $\begin{array}{c}\text { PH } \\
\text { standard } \\
\text { value }\end{array}$ & Temperature $/{ }^{\circ} \mathbf{C}$ & $\begin{array}{c}\text { PH measured } \\
\text { value before } \\
\text { processing }\end{array}$ & $\begin{array}{c}\text { Error before } \\
\text { processing }\end{array}$ & $\begin{array}{c}\text { PH value after } \\
\text { temperature } \\
\text { compensation }\end{array}$ & $\begin{array}{c}\text { Error after } \\
\text { temperature } \\
\text { compensation }\end{array}$ \\
\hline \multirow{3}{*}{4.00} & 10 & 4.11 & +0.11 & 4.03 & +0.03 \\
\cline { 2 - 6 } & 20 & 4.03 & +0.08 & 3.98 & -0.02 \\
\hline \multirow{3}{*}{6.86} & 30 & 3.97 & -0.03 & 4.00 & 0.00 \\
\cline { 2 - 6 } & 10 & 6.96 & +0.01 & 6.86 & 0.00 \\
\hline \multirow{3}{*}{9.18} & 20 & 6.92 & +0.06 & 6.87 & +0.01 \\
\cline { 2 - 6 } & 30 & 6.90 & +0.04 & 6.86 & 0.00 \\
\cline { 2 - 6 } & 10 & 9.06 & -0.12 & 9.20 & +0.02 \\
\hline
\end{tabular}

\section{Conclusion}

Nowadays, the rapid development of wireless sensor networks has become a hot research topic in the field of science and research. This paper is based on the characteristics of PH monitoring network, analysis the design requirements of monitoring nodes, through the in-depth study of types of sensor 
and wireless RF chip, On the basis of CC2530 and Z-Stack protocol stack, an online pH monitoring program based on ZigBee is proposed. Compared with other methods, the method has the advantages of low cost, high data precision, reliability and so on. At the same time, the linear regression algorithm was used to analyze the measurement $\mathrm{pH}$ value, and the temperature compensation model is deduced with the combination of the temperature variation. The experimental results show that the effect of temperature on $\mathrm{PH}$ monitoring was eliminated after the temperature was processed with temperature compensation model.

\section{References}

1. Standards for drinking water quality [EB/OL], [2016-3-20]. http://baike.so.com/doc/53995585637074. html

2. Zhong Jiang, Dan Liu. ZigBee technology and realization course [M]. Beijing: Tsinghua University press(2014)

3. Xiaoqiang Wang, Ouyang Jun, Linning Huang. ZigBee wireless sensor network design and implementation [M]. Beijing: Chemical Industry Press(2012)

4. Shu Wang, Yujie Yan, Fuping Hu. Theory and application of wireless sensor networks [M]. Beijing: Beijing Aeronautics and Astronautics University Press(2007)

5. Chun Jin, Zuqiu Luo. ZigBee technical foundation and case analysis [M]. Beijing: National Defence Industry Press(2008)

6. Xiaowen Chen, Xinde Bai, Xiang Xue. Potential pH equilibrium diagram and its application in corrosion studies of nuclear materials [J]. Tsinghua University Journal, 42 ,5 (2002)

7. Yao Chen, Yueju Xue.PH sensor temperature compensation model [J]. Journal of sensing technology, 25,8(2012) 